\title{
Erratum to: Life Course Influences on Inequalities in Later Life: Comparative Perspectives
}

\author{
Hal Kendig ${ }^{1} \cdot$ James Nazroo $^{2}$
}

Published online: 4 February 2016

(C) Springer Science+Business Media Dordrecht 2016

\section{Erratum to: Population Ageing \\ DOI 10.1007/s12062-015-9138-7}

The original version of this article unfortunately did not include the Acknowledgement. The Acknowledgement should read as below.

We wish to acknowledge generous support from the Oxford Institute on Ageing and the expertise of Kate Hamblin and Anne Mackintosh in liaising with authors and in the production process. The ARC Centre of Excellence in Population Ageing (CEPAR) supported travel by contributors to our invited symposium at the International Sociology Association in Yokohama Japan in 2014. Anonymous referees contributed significantly in improving the work.

The online version of the original article can be found at http://dx.doi.org/10.1007/s12062-015-9138-7.

James Nazroo

james.nazroo@manchester.ac.uk

1 Centre for Research on Ageing, Health, and Well-being, Research School of Population Health, Australian National University, Canberra, Australia

2 Sociology and MICRA, School of Social Sciences, University of Manchester, Manchester, UK 